\title{
Circular RNAs Regulate Cancer Onset and Progression via Wnt/ $\beta$-Catenin Signaling Pathway
}

\author{
Yun-Feng Li, Jian Zhang, and Lei Yu \\ Department of Radiotherapy, The Second Hospital of Jilin University, Changchun, China.
}

\begin{abstract}
Cancer remains to be a major challenge for public health providers, and is the second leading cause of death worldwide. Therefore, it is imperative to explore the mechanisms underlying cancer initiation and development, and design novel diagnostics and therapeutics. Circular RNAs (circRNAs), which exhibit a covalently closed loop structure, are involved in a variety of diseases, including cancer. The aberrant expression of circRNAs contributes to the initiation and development of various cancers by disrupting the interplay of specific signaling pathways, including the Wnt/ $\beta$-catenin pathway, which controls a plethora of cellular processes that drive cancer development. The interactions between circRNAs (specifically expressed in different cancer tissues) and Wnt/ $\beta$-catenin signaling pathway presents potential diagnostic biomarkers and novel therapeutic targets. In this review, we have summarized research discoveries on the functions of Wnt/ $\beta$-catenin pathway-related circRNAs in the modulation of oncogenesis and progression of different types of cancer. We anticipate that our findings will contribute to the improvement or development of circRNAs-based strategies for cancer treatment.
\end{abstract}

Key Words: Circular RNA, cancer, Wnt/ $\beta$-catenin signaling pathway

\section{INTRODUCTION}

Over the past few decades, there have been tremendous advances in cancer therapy, such as chemotherapy, radiotherapy, surgery, targeted therapy, and immunotherapy using immune checkpoint inhibitors. However, cancer has remained a primary public health challenge around the world. ${ }^{1,2}$ In developed countries, the high early detection rate of human cancer has significantly improved the survival rate of patients with different cancer types. However, early detection still remains to be a challenge, especially in developing countries, and cancers detected at advanced stages are difficult to treat, leading to a low survival rate. Therefore, there is an urgent need to explore mechanisms underlying the onset and development of cancer, in or-

Received: August 8, 2019 Revised: October 4, 2019

Accepted: October 15, 2019

Corresponding author: Lei Yu, PhD, Department of Radiotherapy, The Second Hospital of Jilin University, 218 Ziqiang Street, Nanguan, Changchun 130041, China. Tel: 86-0431-81136179, Fax: 86-0431-81136179, E-mail: yul0826@163.com

-The authors have no potential conflicts of interest to disclose.

(C) Copyright: Yonsei University College of Medicine 2019

This is an Open Access article distributed under the terms of the Creative Commons Attribution Non-Commercial License (https://creativecommons.org/licenses/ by-nc/4.0) which permits unrestricted non-commercial use, distribution, and reproduction in any medium, provided the original work is properly cited. der to develop more effective cancer diagnostic and treatment strategies to improve the prognosis and quality of life of cancer patients. $^{3}$

For many years, researchers have focused on the exploration of protein-encoding genes, while noncoding RNA (ncRNA) were ever deemed as "junk" of transcription. ${ }^{4}$ Substantial biological functions of ncRNAs have been observed in recent cancer studies. ${ }^{5,6}$ For instance, long noncoding RNA (lncRNA) NEAT1 promotes nasopharyngeal carcinoma progression via Wnt/ $\beta$-catenin signaling pathway. ${ }^{7}$ Circular RNAs (circRNAs) are a type of endogenous ncRNAs that are first observed and characterized by a covalently closed loop structure, which makes them more stable and with various lengths. ${ }^{8}$ However, some studies have shown that the translation of circRNAs and the category of circRNAs should be re-classified. ${ }^{9,10}$ CircRNAs are steadily expressed in various cells and body fluid; are tissue- and disease-specific; and have unique exon sequences, miRNA response elements, and protein-binding elements. ${ }^{11}$ The unique characteristics of circRNAs indicate that they may be involved in the controlled regulation of cellular biological behaviors. Based on these characteristics, circRNAs have received increased attention, and a variety of functional circRNAs have been reported. circRNAs has been reported to act as a "sponge" for miRNA which consequently modulates the onset and development of vari- 
ous diseases, including cancer. ${ }^{12-15}$ Chen, et al. ${ }^{16}$ found that circPVT1 promoted the proliferation of cancer cells by sponging miR-125. circRNAs can also regulate the progression of cancer through many other mechanisms. For instance, circRNAs can modulate gene transcription and protein translation, activate or inactivate cancer-related signaling pathways, and regulate epithelial-mesenchymal transition (EMT), which is highly associated with the invasion and migration of cancer cells. ${ }^{17-19}$ Through these mechanisms, circRNAs can modulate cancer cells' apoptosis, proliferation, migration, invasion, and angiogenesis. circRNA_100269 has been reported to inhibit the proliferation of gastric cancer (GC) cells by sponging miR-630. ${ }^{20}$ Circ-ZNF652 promotes hepatocellular carcinoma (HCC) metastasis in an EMT-induced manner. ${ }^{21}$ Recent studies have confirmed that circRNAs are involved in various cellular processes, and that they play crucial roles in the initiation and progression of cancer. Therefore, circRNAs can be used for clinical applications, having roles such as diagnostic biomarkers, prognostic biomarkers, and therapeutic targets.

Wnt signaling pathway was discovered in a 1982 study on oncogenic mechanisms using a mouse model, and the first gene to be discovered in the pathway was INT1. ${ }^{22}$ Wnt signaling pathway is divided into $\mathrm{Wnt} / \beta$-catenin-dependent pathway, also known as the canonical pathway, and $\beta$-catenin-independent pathway, which is also divided into Wnt/Jun N-terminal kinase (JNK) pathway and Wnt/Calcium pathway. ${ }^{23,24}$ Canonical pathway has been better understood and characterized, since signal transduction from extracellular liquid to the nucleus was achieved mainly through the accumulation and transfer of $\beta$-catenin protein. ${ }^{25}$ In the absence of Wnt ligand, cytoplasmic $\beta$-catenin protein level remains low, and $\beta$-catenin is recruited into a destruction complex comprising of Axin2, casein kinase1 (CK1), adenomatous polyposis coli (APC), and glycogen synthase kinase $3 \mathrm{~b}$ (GSK3b). ${ }^{25}$ The underlying mechanism here is that the activated CK1 and GSK3b phosphorylate Axin-bound $\beta$-catenin. Subsequently, the phosphorylated $\beta$-catenin is degraded by $26 \mathrm{~S}$ proteasome pathway, aided by E3 ubiquitin ligase such as $\beta$-TrCP. ${ }^{26}$ When Wnt ligands are enough to bind to Frizzled (FZD) receptors and coreceptor lipoprotein-related protein (LRP), disheveled (Dsh, also termed Dvl) protein is activated, which phosphorylates low-density lipoprotein receptor-related protein 5/6 (LRP5/6). Subsequently, the destruction complex is separated by phosphorylated LRP5/6, which occurs through the binding of GSK3b, Axin, and phosphorylated LRP5/6. ${ }^{25,27,28}$ Following the accumulation of $\beta$-catenin in the cytoplasm, it is transferred to the nucleus; and here, it interacts with T cell factor (TCF)/Lymphoid Enhancer Factor (LEF) transcription factors and promotes the transcription of downstream targets, such as cyclin D1, c-Myc, and matrix metalloproteinase $1 .^{29}$

Besides the canonical pathway, several non-canonical Wnt signaling pathways exist, including Wnt/planar cell polarity (PCR) and $\mathrm{WNT} / \mathrm{Ca}^{2+}$ pathways. Wnt/PCR signaling pathway activates
Rho-kinase and JNK cascades. Wnt/ $\mathrm{Ca}^{2+}$ signaling pathway upregulates the concentration of intracellular calcium and activates calcineurin, protein kinase $\mathrm{C}$ (PKC), or CaMKII pathways. Non-canonical Wnt pathways are complicated, and recent studies have reported that non-canonical pathways may exert regulatory effects by suppressing the canonical Wnt/ $\beta$-catenin signaling pathway ${ }^{30,31}$ Additionally, there is evidence showing that non-canonical Wnt pathways play vital roles in the maintenance of cellular biological behaviors, such as cell proliferation, cell migration, cell polarity, and stem cell maintenance, and that they are also tightly associated with the initiation and progression of various cancers. ${ }^{30,32,33}$ circRNAs have been shown to regulate cancer development by modulating Wnt pathway, which is one of the most canonical signaling pathways in cancer initiation and progression. Other studies have revealed that upregulation of Wnt signaling pathway exists in almost all cancer types and plays a role in promoting cancer development and enhances phenotypes such as proliferation, invasion, tube formation, and migration. ${ }^{34}$

Canonical Wnt/ $\beta$-catenin signaling pathway modulates cancer cells apoptosis, proliferation, invasion, and migration in the regulation of initiation and progression of various cancers. ${ }^{34}$ Over the past few years, several studies have indicated that circRNAs modulate gene expressions at both pre-transcription and posttranscription levels. They have also been shown to interact with signaling molecules to influence signal-dependent cell functions and regulate cancer development via $W n t / \beta$-catenin signaling pathway. ${ }^{35}$ Zhi, et al. ${ }^{9}$ reported that circLgr4 upregulated Wnt/ $\beta$-catenin pathway and promoted invasion and tumorigenesis in colorectal cancer (CRC). These findings provide new insights to improve our understanding of the underlying mechanisms of cancer initiation and progression. Therefore, this study aimed to provide a summary of the latest studies on Wnt/ $\beta$-catenin pathway-related circRNAs in specific cancers (Table 1), and also analyze the regulatory mechanisms (Fig. 1) that can be used for the development of novel therapeutics.

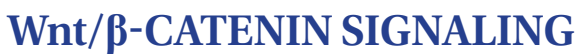 PATHWAY-RELATED circRNAs AND CRC}

CRC is the second most common cause of cancer-related deaths in both males and females worldwide. ${ }^{1}$ A number of studies have reported that differentially expressed circRNAs were observed in CRC tissues compared to the adjacent tissues. This study enumerated recent studies on Wnt/ $\beta$-catenin-related circRNAs and CRC to elucidate the different roles of circRNAs in the onset and development of CRC via the Wnt/ $\beta$-catenin cascade.

Studies have shown that circRNAs acts as a "sponge" for miRNAs to exert regulatory effects in CRC. Downregulation of miRNAs causes circRNAs to increase the expression of miRNA-targeting genes, some of which are involved in $\mathrm{Wnt} / \beta$-catenin signaling pathway. Some circRNAs exert suppressive effects by this mech- 
近 아군

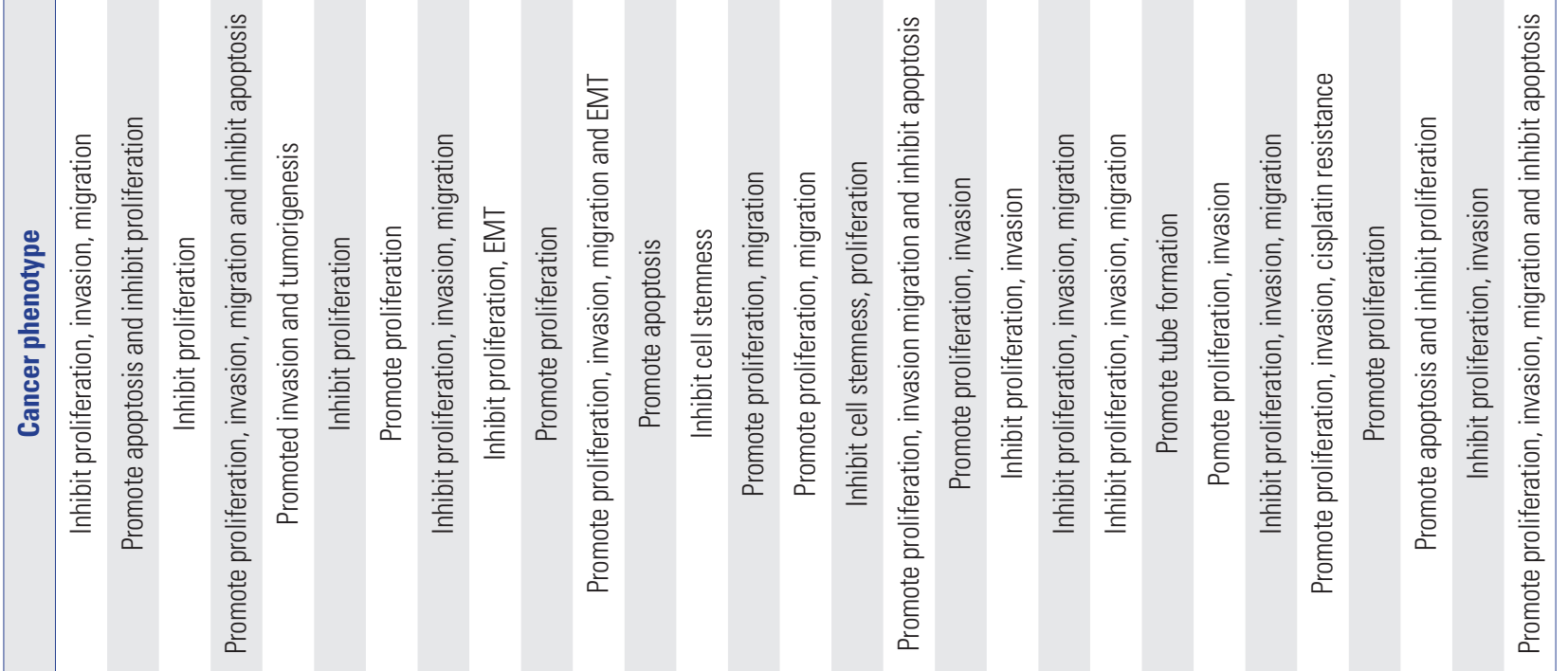

.

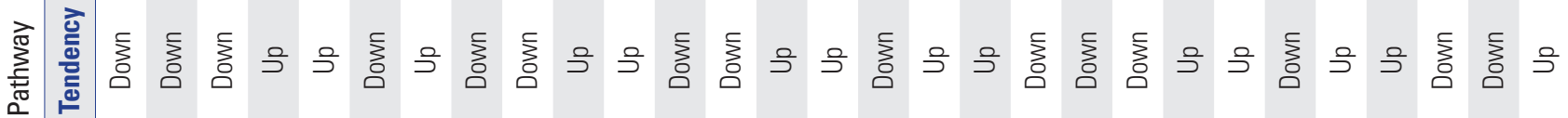
喜



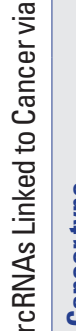

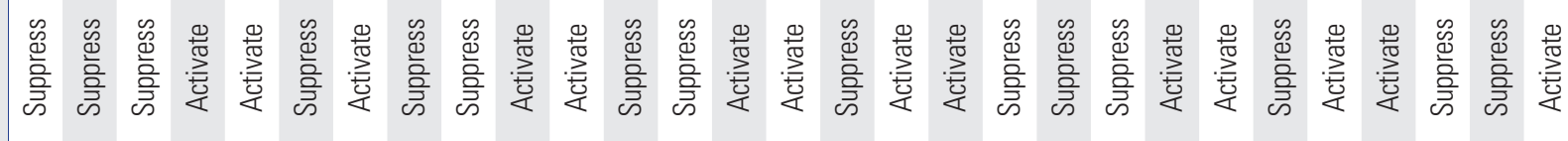<smiles>CCC</smiles> 
anism. ${ }^{36}$ Geng, et al. ${ }^{36}$ noted that hsa_circ_009361 increased APC2 expression and inactivated Wnt/ $\beta$-catenin cascade by sponging miR-582, while miR-582 targeted APC2 mRNA and downregulated its expression. APC is a classic inhibitor in the activation of $W n t / \beta$-catenin cascade, where it forms the destruction complex of $\beta$-catenin. ${ }^{37}$ APC knockdown contributes to the accumulation of $\beta$-catenin in the cytoplasm and transfer to the nucleus. Increasing $\beta$-catenin in the nucleus enhances its interaction with TCF/LEF or other transcription factor coregulators, and modulates downstream target genes involved in cell apoptosis, proliferation, migration, and invasion. ${ }^{25,38,39}$ APC2, a ho- mologous product of APC, suppresses the activation of Wnt/ $\beta$-catenin pathway and leads to the inhibition of CRC cell proliferation, invasion, and migration. In this case, hsa_circ_009361 acts as an endogenous competitive RNA that upregulates the level of APC2. 3'UTR of Dkk1 is closely linked to miR-3, allowing the level of Dkkl protein and mRNA to be upregulated according to the tight binding between has_circ_0000523 and miR-31. ${ }^{40} \mathrm{Dkk} 1$ is a suppressor of Wnt/ $\beta$-catenin pathway, and it inactivates this pathway by binding to LRP5/6 and abolishing its interaction with FZD. ${ }^{41}$ Has_circ_0000523 promotes CRC cells apoptosis and suppresses proliferation via upregulation

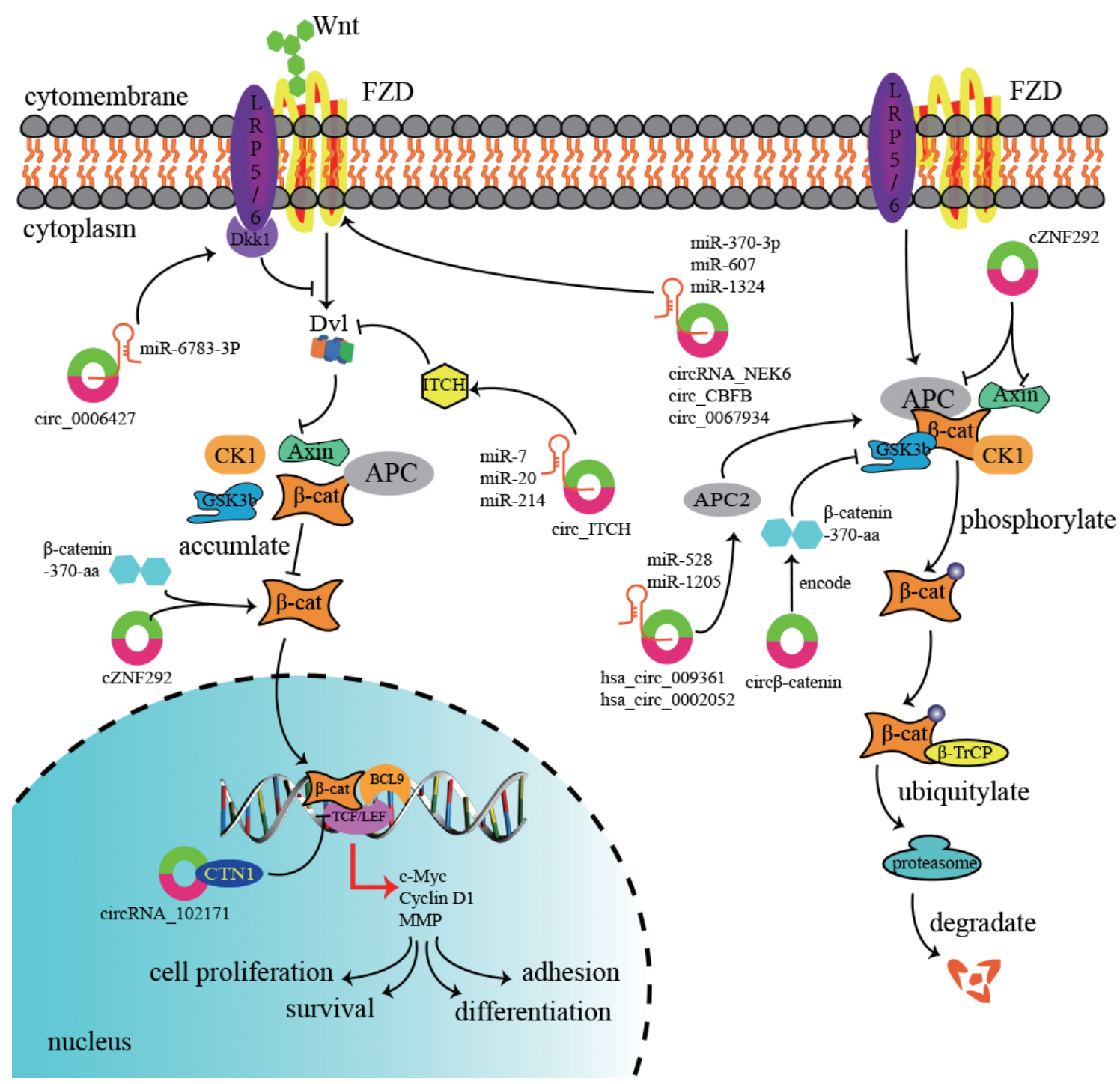

Fig. 1. Diverse regulatory mechanisms of circRNAs and Wnt/ $\beta$-catenin signaling pathway. Circ_0006427 inactivates Wnt/ $\beta$-catenin pathway by upregulating Dkk1 in a miR-6783-3p-dependent manner; Circ_ITCH may increase the expression of protein ITCH by binding to miR-7, miR-20, and miR-124. Subsequently, overexpressed ITCH downregulates Dvl, which reduces $\beta$-catenin degradation and activates this pathway. Three circRNAs (circRNA_NEK6, circ_CBFB and circ_0067934) upregulate frizzled (FZD) expression by sponging corresponding miRNAs (miR-370-3p, miR-607 and miR-1324), and thereby activating the pathway. Hsa_circ_009361 and hsa_circ_0002052 also increase APC2 expression by sponging miR-582 and miR-1205, which inactivates Wnt $/ \beta$-catenin cascade. Circ $\beta$-catenin encodes a $\beta$-catenin isoform, termed " $\beta$-catenin-370-aa", to enhance the stability of $\beta$-catenin by reducing GSK3 $\beta$-induced $\beta$-catenin phosphorylation and degradation, and thereby activating Wnt/ $\beta$-catenin signaling pathway. CircRNA cZNF292 directly upregulates the expression of $\beta$-catenin, thereby activating Wnt/ $\beta$-catenin signaling pathway, but it also suppresses some inhibitors of this pathway, such as APC and Axin. CircRNA_102171 reduces the level of CTNNBIP1 in the nucleus, and enhances the association between $\beta$-catenin and TCF/LEF to upregulate $\mathrm{Wnt} / \beta$-catenin signaling pathway. 
of Dkk1. cir-ITCH plays an anti-tumor role in CRC by sponging miR-214, which binds to the 3' UTR of ITCH mRNA. ITCH protein can ubiquitinate the phosphorylated Dvl2 and facilitates its degradation, whereas cir-ITCH suppresses $W n t / \beta$-catenin signaling pathway and leads to the inhibition of CRC cells proliferation via upregulation of ITCH. ${ }^{42}$ Additionally, in this study, miR-7 and miR-20a were also measured as targets of cir-ITCH to modulate the expression level of ITCH, but statistical analysis of our results showed no statistical significance. Proliferation was the most affected cell phenotypes in CRC cells.

Some circRNAs have been shown to activate this pathway and promote the oncogenesis of CRC. FZD is a vital promotive regulator in the activation of Wnt/ $\beta$-catenin signaling pathway. ${ }^{43,44}$ There are direct binding sites between the 3'UTR of FZD4 and miR-516b, while CircRNA_100290 is able to sponge miR-516b. Therefore, circRNA_100290 overexpression upregulates FZD4 protein level in a miR-516b-dependent manner, and also activates Wnt/ $\beta$-catenin pathway and promote CRC cells proliferation, invasion, and migration, while suppressing apoptosis. ${ }^{45}$

CircRNAs are regarded as lacking protein-encoding ability. Zhi, et al. ${ }^{9}$ reported that circLgr4 encodes a type of peptide to activate Lgr4 protein, upregulates Wnt/ $\beta$-catenin pathway, and promotes the invasion and tumorigenesis in CRC stem cells. Moreover, the knockdown of circLgr4 or Lgr4 impaired Wnt/ $\beta$-catenin activation and decreased CRC stem cells in tumor, hence circLgr4-peptide-Lgr4 pathway can be used for CRC therapy. Previous studies demonstrated that Rspo/Lgr $4 / 5$ signaling pathway is a noncanonical pathway that activates Wnt/ $\beta$-catenin cascade.$^{46}$ Although the underlying mechanisms remain to be explored, Kim, et al. ${ }^{47}$ indicated that RSpo family members activated Wnt/ $\beta$-catenin cascade by binding to Dkk1 and enhancing the activity of LRP6 and Wnt. In combination with Lgr receptors such as Lgr4, Lgr5, and Lgr6, the promotive effect of RSpo in Wnt/ $\beta$-catenin pathway is strengthened..$^{48}$ Additionally, Lgr4 has been reported to be a global marker of cancer stem cells. ${ }^{49}$ This finding contributes not only to our understanding of circRNAs in cancer, but also suggests a novel approach that can be utilized to block Wnt/ $\beta$-catenin activation and inhibit cancer development.

 PATHWAY-RELATED circRNAs AND LUNG CANCER}

Lung cancer is the most commonly diagnosed cancer and the leading cause of cancer-related deaths worldwide. ${ }^{1}$ In 2017, lung cancer, along with stroke and ischemic heart disease, were the three leading causes of deaths in China. ${ }^{50}$ Although great progress has been made with therapeutic strategies, especially targeted therapies, more needs to be done as the prognosis still remains poor. Therefore, there is an urgent need to explore better and effective clinical biomarkers and novel therapeutic targets.
circRNAs have been shown to exert modulatory effects through circRNA-miRNA approach in lung cancer. Wei, et al ${ }^{51}$ reported that significantly decreased expression of cir-ITCH was observed in lung cancer tissues compared to the control, and that important cancer-suppressive effects of cir-ITCH were also found in A549 and NIC-H460 lung cancer cell lines. cir-ITCH exerts anti-tumor effects by sponging miR-7 and miR-214, which bind to the 3' UTR of ITCH mRNA, and causes an increase in the level of E3 ubiquitin ligase ITCH. ITCH binds to Dvl and ubiquitinate it, where it promotes its degradation..$^{51}$ Downregulation of Dvl causes cir-ITCH to block Wnt/ $\beta$-catenin signaling pathway and inhibit the proliferation of lung cancer cells. ${ }^{52}$ Previous studies have indicated that Sirtuin 1 (SIRT1) exerts promotive effects on Wnt/ $\beta$-catenin cascade, ${ }^{53,54}$ miR-135a-5p binds to 3'UTR of SIRT1, and decreases its expression. Based on the interaction between miR-135a-5p and SIRT1, circ_001946 upregulates SIRT1 expression in a miR-135a-5p-dependent manner and activates Wnt/ß-catenin signaling pathway in A549 and H1299 lung cancer cell lines. circ_001946 promotes the proliferation of lung cancer cells.${ }^{55}$ circ_0006427 suppresses lung cancer cell invasion, proliferation, and migration by sponging miR-6783-3p, and also upregulates the expression level of Dkkl and inactivates Wnt/ $\beta$-catenin signaling pathway ${ }^{56}$ In addition, increasing the expression of hsa_circ_0007059 causes a reduction in the level of Wnt3a and $\beta$-catenin in A549 and H1975 lung cancer cells, and thereby inhibiting the activation of Wnt/ $\beta$-catenin cascade. Furthermore, the suppressive effect of overexpressed hsa_circ_0007059 in $\beta$-catenin and Wnt3a is reversed by the transfection of a miR-378 mimic. ${ }^{57}$ Under normal circumstances, target miRNA is negatively associated with the expression of the affected protein. However, in this study, the level of Wnt3a and $\beta$-catenin was positively related to the expression of miR-378, but the underlying mechanisms should be further explored. These findings suggest that hsa_circ_0007059 overexpression attenuates Wnt/ $\beta$-catenin pathway by the suppression of miR-378 in A549 and H1975 lung cancer cells. However, the mechanisms underlying the interactions between miRNAs and their target proteins need to be further explored..$^{57}$

Although the interactions between circRNAs and miRNAs have been widely studied, some circRNA-miRNA interactions still remain unclear. Apart from the circRNA-miRNA approach, circRNAs regulates Wnt/ $\beta$-catenin pathway and cancer development by directly modulating gene transcription, protein translation, and other mechanisms. circ_001569 overexpression upregulates the expression of TCF4, Wntl, and $\beta$-catenin, leading to the activation of Wnt/ $\beta$-catenin pathway and increased proliferation of A549 and H1299 lung cancer cells. ${ }^{58}$ circRNA hsa circ_000984 promotes the activation of Wnt $/ \beta$-catenin signaling pathway as well as the invasion, proliferation, migration, and EMT of non-small cell lung cancer cells, although its direct targets are unknown..$^{59}$

Due to the regulatory functions of circRNAs, there is promising potential in their clinical application for the treatment of lung 
cancer. For example, hsa_circ_0043256 restoration has been utilized in cinnamaldehyde treatment for non-small cell lung cancer. After utilizing cinnamaldehyde, hsa_circ_0043256 is overexpressed and upregulates Wnt/ $\beta$-catenin-suppressor ITCH by sponging miR-1252 while miR-1252 targets ITCH. Therefore, hsa_circ_0043256 inhibits Wnt/ $\beta$-catenin pathway and exerts an anti-tumor effect. ${ }^{60}$ Although effective clinical applications of Wnt/ $\beta$-catenin signaling pathway-related circRNAs are lacking, this suggests a novel therapeutic target that may block or eradicate lung cancer.

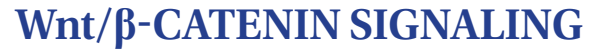 PATHWAY-RELATED circRNAs AND LIVER CANCER}

In China, liver cancer was reported as the fifth most common cause of death in $2017 .{ }^{50} \mathrm{Wnt} / \beta$-catenin cascade, a key regulator of liver cancer development, plays a crucial role in the stemness of cancer cells. ${ }^{61}$ Common markers of liver cancer stemness include CD133, CD90, CD44, and EPCAM. Cell cycle and apoptosis regulator 1 (CCAR1) function as promoters of Wnt/ $\beta$-catenin signaling pathway. Additionally, fragile X mental retardation protein (FMRP) interacts with CCAR1 mRNA and upregulates its expression. In HCC, increasing the expression of circZKSCAN1 inhibits Wnt/ $\beta$-catenin signaling pathway, and suppresses the malignant behavior of HCC by reducing the expression level of FMRP and CCAR1 ${ }^{62}$ In addition, circZKSCAN1 has been shown to be a favorable prognostic indicator of HCC patients. ${ }^{62}$ Therefore, modulating the expression levels of some circRNAs can inhibit cancer cell stemness and block liver cancer progression.

Apart from cell stemness, frequent metastasis and lack of effective chemotherapeutics are tightly associated with poor prognosis in HCC. ${ }^{63,64}$ Therefore, developing effective therapeutic targets is a critical concern for improving the prognosis of HCC patients. circ_0067934 overexpression increases the expression of FZD5 by sponging miR-1324, and this causes the activation of Wnt/ $\beta$-catenin cascade in an FZD5-induced manner. ${ }^{65}$ The activated pathway promotes HCC growth and metastasis. Previous studies have indicated that some circRNAs possess amino acids-encoding capacity. Liang, et al ${ }^{66}$ reported that $\operatorname{circ} \beta$ catenin has the ability to encode a $\beta$-catenin isoform, which contains 370 amino acids and is termed " $\beta$-catenin-370-aa." In addition, $\beta$-catenin-370-aa enhances the stability of $\beta$-catenin by reducing GSK3 $\beta$-induced $\beta$-catenin phosphorylation and degradation, which further activates Wnt/ $\beta$-catenin signaling pathway and promotes liver cancer cell proliferation and migration. ${ }^{66}$ There are some evidence to suggest that circ-ITCH regulates the progression of cancer by modulating Wnt/ $\beta$-catenin signaling pathway. Guo, et al. ${ }^{67}$ reported that circ-ITCH is highly associated with the carcinogenesis of HCC. Additionally, high expression of circ-ITCH indicates better survival rate for HCC patients. Whether circ-ITCH exerts anti-oncogenic effect through Wnt/ $\beta$-catenin pathway in this study needs to be explored further. Therefore, circRNAs molecules exert indispensable functions in cell stemness and proliferation of liver cancer cells, and they have the potential to act as therapeutic targets in clinical treatment.

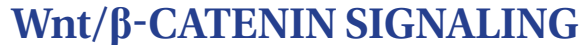 PATHWAY-RELATED circRNAs AND THYROID CANCER}

Although thyroid cancer has been ranked as the ninth leading cause of cancer-related deaths, an estimated 567000 cases have been reported worldwide. ${ }^{1}$ In Korea, it is the most frequently reported tumor, especially in women, at a rate that is 5.5 times higher compared to men. ${ }^{1,68}$ Advances in the treatment of thyroid cancer have significantly improved its prognosis; however, its etiology remains unknown, with ionizing radiation being the only known risk factor. Therefore, exploring the underlying mechanisms is crucial for improving the lives of thyroid cancer patients. CircRNAs are novel regulators in cancer development, with increased attention on their roles in thyroid cancer. For example, circNUP213 has been shown to promote papillary thyroid cancer cells' proliferation, invasion, and migration by sponging miR-145, and thereby upregulating the expression of zinc finger E-box binding homeobox 2 (ZEB2) ${ }^{69}$ circRNA_102171 and circRNA_NEK6 exert promotive functions in thyroid cancer via $\mathrm{Wnt} / \beta$-catenin signaling pathway, while circ-ITCH plays an inhibitory role. ${ }^{70-72}$

CTNNBIP1, a negative regulator of Wnt/ $\beta$-catenin pathway, attenuates the interaction between $\beta$-catenin and TCF/LEF to suppress the expression of downstream targets. ${ }^{73} \mathrm{Bi}$, et al. ${ }^{70}$ reported that circRNA_102171 reduces the level of CTNNBIP1 by interacting with it in the nucleus, and thereby enhancing the association between $\beta$-catenin and TCF/LEF and upregulation of Wnt/ $\beta$-catenin Signaling pathway in papillary thyroid cancer. Therefore, circRNA_102171 promotes thyroid cancer cell proliferation, invasion, and migration, and inhibits apoptosis. Increasing the expression of circRNA_NEK6 upregulates the level of FZD8 by sponging miR-370-3p and activating Wnt/ $\beta$-catenin pathway in thyroid cancer cells. The activated pathway promotes cell proliferation and invasion. ${ }^{71}$ Shashar, et al. ${ }^{74}$ indicated that, apart from CTNNBIP1 and APC, casitas B-lineage lymphoma (CBL) is also an independent negative regulator of $\beta$-catenin, since CBL promotes the degradation of $\beta$-catenin. In addition, MiR-22-3p, a target of circ-ITCH, binds to 3'UTR of CBL mRMA. In papillary thyroid cancer, circ-ITCH overexpression upregulates the expression of CBL in a miR-22-3p-dependent manner, while circ-ITCH suppresses $\mathrm{Wnt} / \beta$-catenin signaling pathway and exerts anti-tumor function. ${ }^{72}$ 


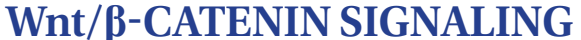 PATHWAY-RELATED circRNAs AND BREAST CANCER}

Breast cancer is the most common cause of cancer-related deaths in females worldwide. ${ }^{1}$ The survival rate of breast cancer patients has improved in recent years, due to the newly developed targeted therapies. Improved understanding of the molecular and genetic alteration underlying breast cancer has led to a more targeted and personalized approach for breast cancer treatment. Despite advances in therapies, prognosis remains poor due to the frequent distal metastasis and advanced diagnosis. The critical regulatory effects of circRNAs identified in cancer development and their interactions with $\mathrm{Wnt} / \beta$-catenin cascade are emerging concerns related to breast cancer. Previous studies have reported that ITCH is a classic inhibitor of Wnt $/ \beta$-catenin signaling pathway. ${ }^{75}$ Downregulated circ-ITCH was observed in triple-negative breast cancer, and further studies revealed that circ-ITCH had the ability to inactivate Wnt/ $\beta$-catenin signaling pathway by sponging miR-214 and miR-17. Upregulation of protein ITCH was reported to facilitate the ubiquitination and degradation of phosphorylated Dvl. Subsequently, downstream genes such as Axin, c-Myc, and cyclin D1 were inhibited, while the proliferation, invasion, and migration of breast cancer cells attenuated. ${ }^{76}$ Therefore, upregulation of circ-ITCH is an effective measure to inhibit the growth and progression of breast cancer, and to further reduce the frequent metastasis and improve prognosis.

\section{Wnt/ק-CATENIN SIGNALING PATHWAY-RELATED circRNAs AND GLIOMA}

Glioma is the most frequently diagnosed brain cancer, and it is responsible for $80 \%$ of malignant brain tumors. ${ }^{77,78}$ In recent years, the roles of signal molecule-related circRNAs in glioma have received increased attention, due to their crucial biological effects. Several circRNAs have been reported to be involved in modulating migration, tube formation, invasion, and proliferation in glioma through $\mathrm{Wnt} / \beta$-catenin signaling pathway. For example, cir-ITCH overexpression was reported to be upregulated in mRNA and protein level of ITCH by serving as a sponge of miR-214 in glioma cells. ${ }^{79}$ Subsequently, increased ITCH suppressed Wnt/ $\beta$-catenin pathway, leading to the inhibition of glioma cell proliferation, invasion, and migration. In addition, upregulating the expression of cir-ITCH inhibited TCF transcriptional activity in glioma cells. Therefore, cir-ITCH plays an anti-oncogenic role in glioma by sponging miR-214 and increasing ITCH expression. Increasing the expression of cir-ITCH can be used to block glioma development. CircRNA cZNF292 modulates glioma tube formation through the regulation of the expression levels of $\beta$-catenin, Axin, and APC, among other pro- teins, which are related to the activation of $\mathrm{Wnt} / \beta$-catenin pathway. Yang, et al ${ }^{80}$ noted that cZNF292 silencing can downregulate the expression of $\beta$-catenin, Cyclin $\mathrm{A}$, and CDK2, which in turn promotes $W n t / \beta$-catenin signaling pathway while increasing some inhibitors of the pathway, such as APC and Axin. Therefore, cZNF292 depletion suppresses glioma progression through the blocking of Wnt/ $\beta$-catenin ${ }^{80}{ }^{80}$ Chen and Duan ${ }^{81}$ identified that hsa_circ_0000177 acts as a "sponge" for miR-638 using bioinformatics analysis. Hsa_circ_0000177 silencing dramatically suppressed the proliferation and invasion of glioma cells in vitro. In addition, miR-638 knockdown restored the invasion and proliferation of glioma cells transfected with hsa_circ_0000177 small interfering RNA. This study identified that FZD7 is a downstream target of miR-638, and is upregulated by increasing the expression of hsa_circ_0000177. Based on these findings, hsa circ_0000177 overexpression promotes malignant behaviors of glioma via FZD7-induced Wnt/ $\beta$-catenin signaling pathway. Therefore, restoring cir-ITCH expression or silencing cZNF292 and hsa_circ_0000177 may provide a potential direction in glioma treatment.

\section{Wnt/ $\beta$-CATENIN SIGNALING PATHWAY-RELATED circRNAs AND OSTEOSARCOMA}

Osteosarcoma (OS) is one of the most common primary malignant bone tumors. Over the past few years, tremendous progress has been made in therapeutic strategies for OS, including surgical resection and chemoradiotherapy. However, the high rate of metastasis remains as a major concern for patients. ${ }^{82}$ Therefore, understanding the molecular mechanisms underlying the initiation and progression of OS is important for OS treatment.

There has been increased attention on the role of circRNAs in OS. Upregulating the expression level of has_circ_0002052 has been reported to reduce the level of miR-1205 while miR-1205 binds to the 3'UTR region of APC2, which is a suppressor of Wnt/ $\beta$-catenin pathway. By releasing the suppressive effect of miR1205 on the expression of APC2, has_circ_0002052 inhibits the activation of Wnt/ $\beta$-catenin pathway, and thereby suppressing OS cell proliferation, invasion, and migration. ${ }^{83}$ This study suggested that has_circ_0002052 overexpression is a potential target in blocking the metastasis of OS. Zhang, et al..$^{84}$ indicated that circ_001569 was overexpressed in OS and associated with the prognosis of OS patients. Knockdown of circ_001569 led to detection of $\beta$-catenin expression, which was decreased, and GSK-3 $\beta$ level upregulated, which attenuated the activity of Wnt/ $\beta$-catenin signaling pathway. These findings suggest that has_circ_0002052 promotes malignant behavior of OS via activation of Wnt $/ \beta$-catenin signaling pathway. 


\section{Wnt/ $\beta$-CATENIN SIGNALING PATHWAY-RELATED circRNAs AND OTHER CANCERS}

Recent studies have suggested that circRNAs modulate the onset and progression of chronic lymphocytic leukemia (CLL), esophageal squamous cell carcinoma (ESCC), and endometrial carcinoma via the canonical Wnt pathway ${ }^{85-87}$ Studies have shown that circRNAs modulates the apoptosis, proliferation, invasion, and migration of these cancers. Studies have also revealed that circRNAs plays an important role in the onset and development of GC, which is also strongly associated with Wnt/ $\beta$-catenin signaling pathway. ${ }^{16,88,89}$ However, the Wnt/ $\beta$-catenin signaling pathway-related circRNAs in GC should be further explored.

CLL is the most common leukemia in adults characterized by overgrowth of abnormal, mature $\mathrm{CD}^{+} \mathrm{CD} 23^{+} \mathrm{B}$ cells in the peripheral blood, bone marrow, and secondary lymphoid tissues, and it is caused by dysregulated cell apoptosis and proliferation. ${ }^{90,91}$ Treatment of CLL has been associated with greater progression, and there are new and emerging therapies targeting vital biological pathways. However, the mechanisms underlying the onset and development of CLL need further exploration. Esophageal cancer is the seventh most commonly diagnosed cancer worldwide, and it is ranked sixth in terms of mortality. ${ }^{1}$ Its incidence among men in Eastern Asia are the highest around the world. ESCC is one of the main subtypes of esophageal cancer derived from esophageal epithelial cells. ${ }^{92}$ Over the past few decades, most cancers, including ESCC, have been deemed as genetic diseases. However, the mechanisms underlying the molecular and genetic basis of esophageal cancer still remain unclear. Endometrial cancer is the most common malignant tumor in the Western female reproductive track, and it is normally treated with surgery. Although many novel therapeutic strategies are being used to improve the prognosis of advanced endometrial cancer patients, the prognosis remains poor. ${ }^{93}$ Therefore, there is an urgent need to investigate the molecular mechanisms underlying the pathogenesis of endometrial carcinoma.

Using TargetScan7.1 (http://www.targetscan.org), Xia, et al. ${ }^{85}$ found that there are four binding sites between miR-607 and FZD3, while circ-CBFB can serve as a sponge for miR-607. Previous studies indicated that overexpressed circ-CBFB increased the expression of FZD3 in a miR-607-dependent manner, thereby activating the Wnt/ $\beta$-catenin Signaling pathway and promoting CLL cell proliferation..$^{85}$ Therefore, knocking down of circCBFB may be a potential target in the treatment of CLL. It has been reported that cir-ITCH upregulates the ITCH protein in a miRNA-dependent manner in several cancers, and that cir-ITCH also sponge miR-7, miR-17, and miR-214 to increase the expression of ITCH and exert anti-tumor effects in ESCC..$^{86}$ In addition, has_circ_0002577 has been shown to be highly conserved to sponge miR-197 in endometrial carcinoma ${ }^{87}$ Given that $3^{\prime}$ UTR of CTNND1 mRNA is tightly linked to miR-197, the expression level of CTNND1 mRNA and its corresponding protein cannot be reduced, due to the binding between has_circ_0002577 and miR-197. ${ }^{87}$ When the CTNND1 protein is accumulated, the interaction between $\beta$-catenin and TCF/LEF is attenuated, and the activity of Wnt/ $\beta$-catenin signaling pathway is downregulated. Furthermore, suppression of tumor growth by knockdown of has_circ_0002577 was observed in vivo. ${ }^{87}$ These findings indicate that has_circ_0002577/miR-197/CTNND1 axis is a potential therapeutic target in the treatment of endometrial carcinoma.

In other diverse cancer cell lines, including prostate cancer, GC, colon cancer, breast cancer, cervical cancer, neuroblastoma, and liver cancer, Yang, et al. ${ }^{94}$ showed that circ-CTNNB1, which was predominantly located in the nucleus, was overexpressed and that it cooperated with DEAD-box polypeptide 3 (DDX3). This promoted the interaction between DDX3 and Yin Yang 1 (YY1), and increased the downstream expression of genes. ${ }^{94} \mathrm{Wnt}$, AXIN, and $\beta$-catenin are target genes of $Y Y 1,{ }^{95}$ and circ-CTNNB1/ DDX3/YY1 axis contributes to upregulation of the expression level of these genes, which then activates Wnt/ $\beta$-catenin cascade and promotes cancer growth and invasion. In most cases, circRNAs modulate the expression level of downstream molecules. In this study, circ-CTNNB1 did not change the protein level of DDX3, but enhanced its effect on $\beta$-catenin. This novel mechanism suggests that, in cases where there is an obvious biological function without change in the proteins targeted by circRNAs, other downstream molecules should be analyzed.

\section{PERSPECTIVE}

In recent years, the incidence of cancer and cancer-related mortality have been rising around the world, posing a great challenge to the public health sector. ${ }^{1}$ Specifically, lung cancer and liver cancer are the two leading causes of deaths in China. ${ }^{50} \mathrm{De}$ spite the advances in development of early detection techniques and therapies, the frequent relapse, metastasis, and chemoresistance have remained to be the key factors responsible for poor prognosis of most cancers. Further studies are needed to identify the mechanisms underlying the onset and development of cancers, in order to facilitate the design of more effective diagnostic and prognostic biomarkers as well as novel therapeutic targets for cancer treatment.

CircRNAs, a type of ncRNA that regulate cancer development, have been getting more attention over the past few years. ${ }^{96} \mathrm{Char}-$ acterized by a covalently closed loop structure, circRNAs are stable in the cytoplasm, nucleus, and body fluids. These molecules modulate various cell behaviors, and also affect the initiation and development of cancer ${ }^{96}$ CircRNAs modulates cancer cell proliferation, invasion, migration, and apoptosis at both pre-transcription and post-transcription levels through various mechanisms. Among these mechanisms, acting as a "sponge" for miRNAs to upregulate the expression level of its targets is the most frequent mechanism. Based on the data reviewed in this study, circRNAs can also exert regulatory effects on gene transcription 
and protein expression, and modulate the level of some signaling molecules, such as encoding peptide and amino acids. Additionally, circRNAs also act as "enhancers" that regulate the activity of some proteins, such as circ-CTNNB1, without changing their expression levels. ${ }^{94}$ These regulatory functions make circRNAs important in cancer research. Out of all Wnt $/ \beta$-catenin signaling pathway-related circRNAs, circ-ITCH shows promising prospects. It has been found to be downregulated in glioma, ESCC, CRC, lung cancer, liver cancer, thyroid cancer, and breast cancer. Moreover, there is consensus that overexpression of circITCH exerts anti-tumor effects in an ITCH protein-dependent manner by sponging corresponding miRNAs. ITCH protein is a member of Nedd4-like E3 family, which contains four WW domains related to PPxY-containing targets. The downstream targets of circ-ITCH regulate the progression and chemoresistance of tumors. In addition, several studies have shown that ITCH promotes the degradation of phosphorylated Dvl via $26 \mathrm{~S}$ proteasome, and thereby inactivating Wnt/ $\beta$-catenin signaling pathway. ${ }^{86}$ Canonical Wnt pathway normally promotes cancer development. Therefore, increasing the expression of ITCH may inhibit cancer initiation and progression. Another study reported that 3'UTR of ITCH and circ-ITCH share some miRNAs binding sites, and that circ-ITCH can upregulate the level of ITCH by acting as a "sponge" for these miRNAs. ${ }^{79}$ This way, circ-ITCH exerts anti-tumor effects in an ITCH-dependent manner.

Among the diverse signaling pathways mediating the effects of circRNAs on cellular processes, Wnt/ $\beta$-catenin pathway is a common one in many cancers and plays critical roles in the onset and development these cancers. ${ }^{97}$ By interacting with signaling pathway-related molecules, such as DNA, miRNA, and transcription factors, circRNAs activate or inhibit Wnt/ $\beta$-catenin signaling pathway to impact biological processes. Additionally, circRNAs modulate Wnt/ $\beta$-catenin signaling pathway not only through the classic approach, but also via other bypass pathways, such as Rspo/Lgr4 pathway and CTNNBIP1 pathway. Among the main targeted molecules, CTNNB1, ITCH, APC, and $\mathrm{CBL}$ are negative regulators of $\mathrm{Wnt} / \beta$-catenin signaling pathway, whereas FZD, $\beta$-catenin, and Dvl activate this pathway. These reports indicate that circRNAs considerably affect the pathology of various cancers via Wnt/ $\beta$-catenin pathway. Some Wnt/ $\beta$-catenin-related circRNAs have been summarized in Table 1 . Our results suggest that the interaction between circRNAs and Wnt/ $\beta$-catenin pathway can be a potential target for cancer therapeutics and diagnostic biomarkers.

\section{CONCLUSION}

In summary, Wnt/ $\beta$-catenin pathway-related circRNAs play key roles in different types of cancer. These RNAs regulate downstream targets that are tightly associated with oncogenesis, cancer cell phenotypes, and cancer progression. The interactions between circRNAs and Wnt/ $\beta$-catenin signaling pathway show significant potential for finding novel therapeutic targets and diagnostic biomarkers. The mechanisms underlying such interactions should be further studied to develop combination treatments. Numerous circRNAs have been identified to interact with Wnt/ $\beta$-catenin pathway in many types of cancer by acting as a "sponge" for miRNAs, or by modulating key molecules of this pathway, such as FZD, $\beta$-catenin, and APC. However, some $\mathrm{Wnt} / \beta$-catenin pathway-related circRNAs as well as their functions and targets remain unclear, and need to be explored in the future.

\section{AUTHOR CONTRIBUTIONS}

Conceptualization: Lei Yu. Data curation: Jian Zhang. Formal analysis: Yun-Feng Li. Funding acquisition: Lei Yu. Investigation: Yun-Feng Li. Methodology: Lei Yu. Project administration: Lei Yu. Resources: Yun-Feng Li. Software: Jian Zhang. Supervision: Lei Yu. Validation: Yun-Feng Li. Visualization: Jian Zhang. Writing-original draft: Yun-Feng Li. Writing_-review \& editing: Lei Yu and Yun-Feng Li.

\section{ORCID iDs}

Yun-Feng Li Jian Zhang

Lei Yu https://orcid.org/0000-0003-4988-8373 https://orcid.org/0000-0002-4941-7590 https://orcid.org/0000-0003-0010-9228

\section{REFERENCES}

1. Bray F, Ferlay J, Soerjomataram I, Siegel RL, Torre LA, Jemal A. Global cancer statistics 2018: GLOBOCAN estimates of incidence and mortality worldwide for 36 cancers in 185 countries. CA Cancer J Clin 2018;68:394-424.

2. Chen W, Zheng R, Baade PD, Zhang S, Zeng H, Bray F, et al. Cancer statistics in China, 2015. CA Cancer J Clin 2016;66:115-32.

3. Lu D, Luo P, Wang Q, Ye Y, Wang B. lncRNA PVT1 in cancer: a review and meta-analysis. Clin Chim Acta 2017;474:1-7.

4. Cech TR, Steitz JA. The noncoding RNA revolution-trashing old rules to forge new ones. Cell 2014;157:77-94.

5. Hu X, Feng Y, Zhang D, Zhao SD, Hu Z, Greshock J, et al. A functional genomic approach identifies FAL1 as an oncogenic long noncoding RNA that associates with BMI1 and represses p21 expression in cancer. Cancer Cell 2014;26:344-57.

6. Tang X, Qiao X, Chen C, Liu Y, Zhu J, Liu J. Regulation mechanism of long noncoding RNAs in colon cancer development and progression. Yonsei Med J 2019;60:319-25.

7. Ji Y, Wang M, Li X, Cui F. The long noncoding RNA NEAT1 targets miR-34a-5p and drives nasopharyngeal carcinoma progression via Wnt/ $\beta$-catenin signaling. Yonsei Med J 2019;60:336-45.

8. Rong D, Sun H, Li Z, Liu S, Dong C, Fu K, et al. An emerging function of circRNA-miRNAs-mRNA axis in human diseases. Oncotarget 2017;8:73271-81.

9. Zhi X, Zhang J, Cheng Z, Bian L, Qin J. circLgr4 drives colorectal tumorigenesis and invasion through Lgr4-targeting peptide. Int J Cancer 2019 Jul 3 [Epub]. Available at: https://doi.org.10.1002/ ijc.32549.

10. Sun HD, Xu ZP, Sun ZQ, Zhu B, Wang Q, Zhou J, et al. Down-regulation of circPVRL3 promotes the proliferation and migration of gastric cancer cells. Sci Rep 2018;8:10111.

11. Liu J, Liu T, Wang X, He A. Circles reshaping the RNA world: from 
waste to treasure. Mol Cancer 2017;16:58.

12. Han D, Li J, Wang H, Su X, Hou J, Gu Y, et al. Circular RNA circMTO1 acts as the sponge of microRNA-9 to suppress hepatocellular carcinoma progression. Hepatology 2017;66:1151-64.

13. Tang YY, Zhao P, Zou TN, Duan JJ, Zhi R, Yang SY, et al. Circular RNA hsa_circ_0001982 promotes breast cancer cell carcinogenesis through decreasing miR-143. DNA Cell Biol 2017;36:901-8.

14. Zhang J, Liu H, Hou L, Wang G, Zhang R, Huang Y, et al. Circular RNA_LARP4 inhibits cell proliferation and invasion of gastric cancer by sponging miR-424-5p and regulating LATS1 expression. Mol Cancer 2017;16:151.

15. Wang K, Long B, Liu F, Wang JX, Liu CY, Zhao B, et al. A circular RNA protects the heart from pathological hypertrophy and heart failure by targeting miR-223. Eur Heart J 2016;37:2602-11.

16. Chen J, Li Y, Zheng Q, Bao C, He J, Chen B, et al. Circular RNA profile identifies circPVT1 as a proliferative factor and prognostic marker in gastric cancer. Cancer Lett 2017;388:208-19.

17. Li P, Chen H, Chen S, Mo X, Li T, Xiao B, et al. Circular RNA 0000096 affects cell growth and migration in gastric cancer. $\mathrm{Br} \mathrm{J}$ Cancer 2017;116:626-33.

18. Wang L, Tong X, Zhou Z, Wang S, Lei Z, Zhang T, et al. Circular RNA hsa_circ_0008305 (circPTK2) inhibits TGF- $\beta$-induced epithelial-mesenchymal transition and metastasis by controlling TIF1 $\gamma$ in non-small cell lung cancer. Mol Cancer 2018;17:140.

19. Li Y, Wan B, Liu L, Zhou L, Zeng Q. Circular RNA circMTO1 suppresses bladder cancer metastasis by sponging miR-221 and inhibiting epithelial-to-mesenchymal transition. Biochem Biophys Res Commun 2019;508:991-6.

20. Zhang Y, Liu H, Li W, Yu J, Li J, Shen Z, et al. CircRNA_100269 is downregulated in gastric cancer and suppresses tumor cell growth by targeting miR-630. Aging (Albany NY) 2017;9:1585-94.

21. Guo J, Duan H, Li Y, Yang L, Yuan L. A novel circular RNA circZNF652 promotes hepatocellular carcinoma metastasis through inducing snail-mediated epithelial-mesenchymal transition by sponging miR-203/miR-502-5p. Biochem Biophys Res Commun 2019;513:812-9.

22. Nusse R, Varmus HE. Many tumors induced by the mouse mammary tumor virus contain a provirus integrated in the same region of the host genome. Cell 1982;31:99-109.

23. Zuo Y, Liu Y. New insights into the role and mechanism of Wnt/ $\beta$-catenin signalling in kidney fibrosis. Nephrology (Carlton) 2018;23 Suppl 4:38-43.

24. Kikuchi A, Yamamoto H, Sato A, Matsumoto S. New insights into the mechanism of Wnt signaling pathway activation. Int Rev Cell Mol Biol 2011;291:21-71.

25. Nusse R, Clevers H. Wnt/ $\beta$-catenin signaling, disease, and emerging therapeutic modalities. Cell 2017;169:985-99.

26. Vijayakumar S, Liu G, Wen HC, Abu Y, Chong R, Nastri H, et al. Extracellular LDLR repeats modulate Wnt signaling activity by promoting LRP6 receptor endocytosis mediated by the Itch E3 ubiquitin ligase. Genes Cancer 2017;8:613-27.

27. Mao J, Wang J, Liu B, Pan W, Farr GH 3rd, Flynn C, et al. Low-density lipoprotein receptor-related protein-5 binds to Axin and regulates the canonical Wnt signaling pathway. Mol Cell 2001;7:801-9.

28. Daniels DL, Weis WI. Beta-catenin directly displaces Groucho/ TLE repressors from Tcf/Lef in Wnt-mediated transcription activation. Nat Struct Mol Biol 2005;12:364-71.

29. Valenta T, Hausmann G, Basler K. The many faces and functions of $\beta$-catenin. EMBO J 2012;31:2714-36.

30. Li C, Bellusci S, Borok Z, Minoo P. Non-canonical WNT signalling in the lung. J Biochem 2015;158:355-65.

31. Zhang S, Chen L, Cui B, Chuang HY, Yu J, Wang-Rodriguez J, et al. ROR1 is expressed in human breast cancer and associated with enhanced tumor-cell growth. PLoS One 2012;7:e31127.

32. Sandsmark E, Hansen AF, Selnæs KM, Bertilsson H, Bofin AM, Wright AJ, et al. A novel non-canonical Wnt signature for prostate cancer aggressiveness. Oncotarget 2017;8:9572-86.

33. Yan J, Liu T, Zhou X, Dang Y, Yin C, Zhang G. FZD6, targeted by miR-21, represses gastric cancer cell proliferation and migration via activating non-canonical wnt pathway. Am J Transl Res 2016; 8:2354-64.

34. Krishnamurthy N, Kurzrock R. Targeting the Wnt/beta-catenin pathway in cancer: update on effectors and inhibitors. Cancer Treat Rev 2018;62:50-60.

35. Zhu L, Ren T, Zhu Z, Cheng M, Mou Q, Mu M, et al. Thymosin- $\beta 4$ mediates hepatic stellate cell activation by interfering with CircRNA-0067835/miR-155/FoxO3 signaling pathway. Cell Physiol Biochem 2018;51:1389-98.

36. Geng Y, Zheng X, Hu W, Wang Q, Xu Y, He W, et al. Hsa_circ_0009361 acts as the sponge of miR-582 to suppress colorectal cancer progression by regulating APC2 expression. Clin Sci (Lond) 2019;133: 1197-213.

37. Fodde R, Brabletz T. Wnt/beta-catenin signaling in cancer stemness and malignant behavior. Curr Opin Cell Biol 2007;19:150-8.

38. Anastas JN, Moon RT. WNT signalling pathways as therapeutic targets in cancer. Nat Rev Cancer 2013;13:11-26.

39. Clevers H, Nusse R. Wnt/ $\beta$-catenin signaling and disease. Cell 2012;149:1192-205.

40. Jin Y, Yu LL, Zhang B, Liu CF, Chen Y. Circular RNA hsa_circ_0000523 regulates the proliferation and apoptosis of colorectal cancer cells as miRNA sponge. Braz J Med Biol Res 2018;51:e7811.

41. Lv C, Li F, Li X, Tian Y, Zhang Y, Sheng X, et al. MiR-31 promotes mammary stem cell expansion and breast tumorigenesis by suppressing Wnt signaling antagonists. Nat Commun 2017;8:1036.

42. Huang G, Zhu H, Shi Y, Wu W, Cai H, Chen X. cir-ITCH plays an inhibitory role in colorectal cancer by regulating the Wnt/ $\beta$-catenin pathway. PLoS One 2015;10:e131225.

43. Skronska-Wasek W, Mutze K, Baarsma HA, Bracke KR, Alsafadi $\mathrm{HN}$, Lehmann M, et al. Reduced frizzled receptor 4 expression prevents WNT/ $\beta$-catenin-driven alveolar lung repair in chronic obstructive pulmonary disease. Am J Respir Crit Care Med 2017; 196:172-85.

44. Yang Y, Sun Y, Wu Y, Tang D, Ding X, Xu W, et al. Downregulation of miR-3127-5p promotes epithelial-mesenchymal transition via FZD4 regulation of Wnt/ $\beta$-catenin signaling in non-small-cell lung cancer. Mol Carcinog 2018;57:842-53.

45. Fang G, Ye BL, Hu BR, Ruan XJ, Shi YX. CircRNA_100290 promotes colorectal cancer progression through miR-516b-induced downregulation of FZD4 expression and Wnt/ $\beta$-catenin signaling. Biochem Biophys Res Commun 2018;504:184-9.

46. Carmon KS, Gong X, Lin Q, Thomas A, Liu Q. R-spondins function as ligands of the orphan receptors LGR4 and LGR5 to regulate Wnt/beta-catenin signaling. Proc Natl Acad Sci U S A 2011;108: 11452-7.

47. Kim KA, Wagle M, Tran K, Zhan X, Dixon MA, Liu S, et al. RSpondin family members regulate the Wnt pathway by a common mechanism. Mol Biol Cell 2008;19:2588-96.

48. Wang D, Huang B, Zhang S, Yu X, Wu W, Wang X. Structural basis for R-spondin recognition by LGR4/5/6 receptors. Genes Dev 2013;27:1339-44.

49. Nakata S, Phillips E, Goidts V. Emerging role for leucine-rich repeat-containing G-protein-coupled receptors LGR5 and LGR4 in cancer stem cells. Cancer Manag Res 2014;6:171-80.

50. Zhou M, Wang H, Zeng X, Yin P, Zhu J, Chen W, et al. Mortality, morbidity, and risk factors in China and its provinces, 1990-2017: a systematic analysis for the Global Burden of Disease Study 2017. 
Lancet 2019;394:1145-58.

51. Wei W, Li M, Wang J, Nie F, Li L. The E3 ubiquitin ligase ITCH negatively regulates canonical Wnt signaling by targeting dishevelled protein. Mol Cell Biol 2012;32:3903-12.

52. Wan L, Zhang L, Fan K, Cheng ZX, Sun QC, Wang JJ. Circular RNA-ITCH suppresses lung cancer proliferation via inhibiting the Wnt/ $\beta$-catenin pathway. Biomed Res Int 2016;2016:1579490.

53. Luo Y, Chen JJ, Lv Q, Qin J, Huang YZ, Yu MH, et al. Long noncoding RNA NEAT1 promotes colorectal cancer progression by competitively binding miR-34a with SIRT1 and enhancing the Wnt/ $\beta$-catenin signaling pathway. Cancer Lett 2019;440-441:11-22.

54. Zhou Y, Song T, Peng J, Zhou Z, Wei H, Zhou R, et al. SIRT1 suppresses adipogenesis by activating Wnt/ $\beta$-catenin signaling in vivo and in vitro. Oncotarget 2016;7:77707-20.

55. Yao Y, Hua Q, Zhou Y, Shen H. CircRNA has_circ_0001946 promotes cell growth in lung adenocarcinoma by regulating miR-135a-5p/ SIRT1 axis and activating $W n t / \beta$-catenin signaling pathway. Biomed Pharmacother 2019;111:1367-75.

56. Yao Y, Hua Q, Zhou Y. CircRNA has_circ_0006427 suppresses the progression of lung adenocarcinoma by regulating miR-6783-3p/ DKK1 axis and inactivating Wnt/ $\beta$-catenin signaling pathway. Biochem Biophys Res Commun 2019;508:37-45.

57. Gao S, Yu Y, Liu L, Meng J, Li G. Circular RNA hsa_circ_0007059 restrains proliferation and epithelial-mesenchymal transition in lung cancer cells via inhibiting microRNA-378. Life Sci 2019;233: 116692.

58. Ding L, Yao W, Lu J, Gong J, Zhang X. Upregulation of circ_001569 predicts poor prognosis and promotes cell proliferation in nonsmall cell lung cancer by regulating the Wnt/ $\beta$-catenin pathway. Oncol Lett 2018;16:453-8.

59. Li XY, Liu YR, Zhou JH, Li W, Guo HH, Ma HP. Enhanced expression of circular RNA hsa_circ_000984 promotes cells proliferation and metastasis in non-small cell lung cancer by modulating Wnt/ $\beta$-catenin pathway. Eur Rev Med Pharmacol Sci 2019;23:3366-74.

60. Tian F, Yu CT, Ye WD, Wang Q. Cinnamaldehyde induces cell apoptosis mediated by a novel circular RNA hsa_circ_0043256 in non-small cell lung cancer. Biochem Biophys Res Commun 2017; 493:1260-6.

61. Hoffmeyer K, Raggioli A, Rudloff S, Anton R, Hierholzer A, Del Valle $\mathrm{I}$, et al. Wnt/ $\beta$-catenin signaling regulates telomerase in stem cells and cancer cells. Science 2012;336:1549-54.

62. Zhu YJ, Zheng B, Luo GJ, Ma XK, Lu XY, Lin XM, et al. Circular RNAs negatively regulate cancer stem cells by physically binding FMRP against CCAR1 complex in hepatocellular carcinoma. Theranostics 2019;9:3526-40.

63. Jiang Y, Sun A, Zhao Y, Ying W, Sun H, Yang X, et al. Proteomics identifies new therapeutic targets of early-stage hepatocellular carcinoma. Nature 2019;567:257-61.

64. Villanueva A. Hepatocellular carcinoma. N Engl J Med 2019;380: 1450-62.

65. Zhu Q, Lu G, Luo Z, Gui F, Wu J, Zhang D, et al. CircRNA circ_0067934 promotes tumor growth and metastasis in hepatocellular carcinoma through regulation of miR-1324/FZD5/Wnt/ $\beta$-catenin axis. Biochem Biophys Res Commun 2018;497:626-32.

66. Liang WC, Wong CW, Liang PP, Shi M, Cao Y, Rao ST, et al. Translation of the circular RNA $\operatorname{circ} \beta$-catenin promotes liver cancer cell growth through activation of the Wnt pathway. Genome Biol 2019;20:84.

67. Guo W, Zhang J, Zhang D, Cao S, Li G, Zhang S, et al. Polymorphisms and expression pattern of circular RNA circ-ITCH contributes to the carcinogenesis of hepatocellular carcinoma. Oncotarget 2017;8:48169-77.

68. Oh CM, Kong HJ, Kim E, Kim H, Jung KW, Park S, et al. National
Epidemiologic Survey of Thyroid cancer (NEST) in Korea. Epidemiol Health 2018;40:e2018052.

69. Li X, Tian Y, Hu Y, Yang Z, Zhang L, Luo J. CircNUP214 sponges miR-145 to promote the expression of ZEB2 in thyroid cancer cells. Biochem Biophys Res Commun 2018;507:168-72.

70. Bi W, Huang J, Nie C, Liu B, He G, Han J, et al. CircRNA circRNA_102171 promotes papillary thyroid cancer progression through modulating CTNNBIP1-dependent activation of $\beta$-catenin pathway. J Exp Clin Cancer Res 2018;37:275.

71. Chen F, Feng Z, Zhu J, Liu P, Yang C, Huang R, et al. Emerging roles of circRNA_NEK6 targeting miR-370-3p in the proliferation and invasion of thyroid cancer via Wnt signaling pathway. Cancer Biol Ther 2018;19:1139-52.

72. Wang M, Chen B, Ru Z, Cong L. CircRNA circ-ITCH suppresses papillary thyroid cancer progression through miR-22-3p/CBL/ $\beta$-catenin pathway. Biochem Biophys Res Commun 2018;504: 283-8.

73. Fu X, Zhu X, Qin F, Zhang Y, Lin J, Ding Y, et al. Linc00210 drives Wnt/ $\beta$-catenin signaling activation and liver tumor progression through CTNNBIP1-dependent manner. Mol Cancer 2018;17:73.

74. Shashar M, Siwak J, Tapan U, Lee SY, Meyer RD, Parrack P, et al. c$\mathrm{Cbl}$ mediates the degradation of tumorigenic nuclear $\beta$-catenin contributing to the heterogeneity in Wnt activity in colorectal tumors. Oncotarget 2016;7:71136-50.

75. Lim SK, Lu SY, Kang SA, Tan HJ, Li Z, Adrian Wee ZN, et al. Wnt signaling promotes breast cancer by blocking ITCH-mediated degradation of YAP/TAZ transcriptional coactivator WBP2. Cancer Res 2016;76:6278-89.

76. Wang ST, Liu LB, Li XM, Wang YF, Xie PJ, Li Q, et al. Circ-ITCH regulates triple-negative breast cancer progression through the Wnt/ $\beta$-catenin pathway. Neoplasma 2019;66:232-9.

77. McTyre E, Lucas JT, Helis C, Farris M, Soike M, Mott R, et al. Outcomes for anaplastic glioma treated with radiation therapy with or without concurrent temozolomide. Am J Clin Oncol 2018;41: 813-9.

78. Zhang Y, Yu X, Chen L, Zhang Z, Feng S. EZH2 overexpression is associated with poor prognosis in patients with glioma. Oncotarget 2017;8:565-73.

79. Li F, Ma K, Sun M, Shi S. Identification of the tumor-suppressive function of circular RNA ITCH in glioma cells through sponging miR-214 and promoting linear ITCH expression. Am J Transl Res 2018;10:1373-86.

80. Yang P, Qiu Z, Jiang Y, Dong L, Yang W, Gu C, et al. Silencing of cZNF292 circular RNA suppresses human glioma tube formation via the Wnt/ $\beta$-catenin signaling pathway. Oncotarget 2016;7: 63449-55.

81. Chen Z, Duan X. hsa_circ_0000177-miR-638-FZD7-Wnt signaling cascade contributes to the malignant behaviors in glioma. DNA Cell Biol 2018;37:791-7.

82. Thompson LD. Osteosarcoma. Ear Nose Throat J 2013;92:288-90.

83. Wu Z, Shi W, Jiang C. Overexpressing circular RNA hsa_circ_0002052 impairs osteosarcoma progression via inhibiting Wnt/ $\beta$-catenin pathway by regulating miR-1205/APC2 axis. Biochem Biophys Res Commun 2018;502:465-71.

84. Zhang H, Yan J, Lang X, Zhuang Y. Expression of circ_001569 is upregulated in osteosarcoma and promotes cell proliferation and cisplatin resistance by activating the $\mathrm{Wnt} / \beta$-catenin signaling pathway. Oncol Lett 2018;16:5856-62.

85. Xia L, Wu L, Bao J, Li Q, Chen X, Xia H, et al. Circular RNA circCBFB promotes proliferation and inhibits apoptosis in chronic lymphocytic leukemia through regulating miR-607/FZD3/Wnt/ $\beta$-catenin pathway. Biochem Biophys Res Commun 2018;503: 385-90. 
86. Li F, Zhang L, Li W, Deng J, Zheng J, An M, et al. Circular RNA ITCH has inhibitory effect on ESCC by suppressing the Wnt/ $\beta$-catenin pathway. Oncotarget 2015;6:6001-13.

87. Shen Q, He T, Yuan H. Hsa_circ_0002577 promotes endometrial carcinoma progression via regulating miR-197/CTNND1 axis and activating Wnt/ $\beta$-catenin pathway. Cell Cycle 2019;18:1229-40.

88. Shao Y, Chen L, Lu R, Zhang X, Xiao B, Ye G, et al. Decreased expression of hsa_circ_0001895 in human gastric cancer and its clinical significances. Tumour Biol 2017;39:1010428317699125.

89. Song X, Xin N, Wang W, Zhao C. Wnt/ $\beta$-catenin, an oncogenic pathway targeted by $\mathrm{H}$. pylori in gastric carcinogenesis. Oncotarget 2015;6:35579-88.

90. Zenz T, Mertens D, Küppers R, Döhner H, Stilgenbauer S. From pathogenesis to treatment of chronic lymphocytic leukaemia. Nat Rev Cancer 2010;10:37-50.

91. Bosch F, Dalla-Favera R. Chronic lymphocytic leukaemia: from genetics to treatment. Nat Rev Clin Oncol 2019;16:684-701.

92. Dong S, Zhang P, Liang S, Wang S, Sun P, Wang Y. The role of the retinoblastoma protein-interacting zinc finger gene 1 tumor suppressor gene in human esophageal squamous cell carcinoma cells. Oncol Lett 2013;6:1656-62.

93. Fader AN, Arriba LN, Frasure HE, von Gruenigen VE. Endometrial cancer and obesity: epidemiology, biomarkers, prevention and survivorship. Gynecol Oncol 2009;114:121-7.

94. Yang F, Fang E, Mei H, Chen Y, Li H, Li D, et al. Cis-acting circCTNNB1 promotes $\beta$-catenin signaling and cancer progression via DDX3-mediated transactivation of YY1. Cancer Res 2019;79: $557-71$.

95. Lu C, Thoeni C, Connor A, Kawabe H, Gallinger S, Rotin D. Intestinal knockout of Nedd4 enhances growth of Apc(min) tumors. Oncogene 2016;35:5839-49.

96. Zhao ZJ, Shen J. Circular RNA participates in the carcinogenesis and the malignant behavior of cancer. RNA Biol 2017;14:514-21.

97. Shiah SG, Shieh YS, Chang JY. The role of Wnt signaling in squamous cell carcinoma. J Dent Res 2016;95:129-34. 